\title{
IDENTIFICATION DES FACTEURS DE CRISE MANAGERIALE PROVOQUEE PAR L'ABSENCE DES STRATEGIES DES TIC : CAS DES ENTREPRISES DE LA RDC
}

\author{
Author(s) / Auteur(s) : \\ Rodrigue IYEMBO NGINDA \\ Licencié en Informatique de Gestion \\ Assistant MIS (Management Information System) du \\ Professeur Alphonse Christian IVINZA LEPAPA (Membre S\&O) \\ rodriveille@gmail.com
}

\begin{abstract}
Résumé :
"Les technologies de l'information deviennent de plus en plus l'élément clé du bien-être économique national, car elles touchent à peu près tout ce qui est industrie ou service. On aurait du mal à trouver une fonction ne dépendant pas de l'utilisation efficace de l'information : pour étudier produits et services, pour suivre les demandes de marché et pour y répondre, pour prendre des décisions bien informées.

Les TIC vont changer le monde de façon plus permanente et plus profonde qu'aucune autre technologie ne l'aura jamais fait au cours de l'histoire et nous aurons à nous accommoder au changement de civilisation que cela entraînera." (IVINZA, 2017, p.183)

Est-ce que l'absence de stratégique des TIC est-elle à la base de certaines crises managériales en RDC?

L'approche stratégique des TIC est utilisée dans les organisations pour appuyer ou porter la stratégie concurrentielle, et gagner ou maintenir un avantage concurrentiel qui nécessite pour le dirigeant d'adhérer à trois principes fondamentaux :

$\checkmark \quad$ La définition de ce qu'est une entreprise,

$\checkmark \quad$ L'utilisation des ressources de l'entreprise,

$\checkmark \quad$ Le rôle de l'entrepreneur comme agent du changement dans l'entreprise.

L'adoption des TIC est à la base des certaines crises managériales des entreprises en RDC, Tandis que le fondement managérial de l'approche classique est basé sur l'économie des coûts dont certains managers $n$ 'ont pas la maitrise. Les managers congolais confondent deux paramètres qui affectent leurs actions dans l'entreprise et prennent parfois l'un à la place de l'autre : il s'agit du risque et de l'incertitude. (COBBAUT, 1994, p.52)
\end{abstract}

Keywords / Mots-clés:

TIC, crise managériale, approche stratégique, risque, incertitude

Dans cet article, nous aborderons d'abord les concepts liés à la crise, stratégie, et au management de systèmes d'information. Ensuite, nous présenterons l'approche managériale et l'adoption des TIC dans la gestion des entreprises. Enfin nous identifierons certains facteurs de crise managériale provenant de l'absence de l'approche stratégique des TIC en Afrique et en RD Congo.

\section{CONCEPT DE CRISE}

Les avènements de crise se caractérisent par des situations imprévisibles et pleines de difficultés ${ }^{1}$; des moments d'incertitude et de perturbation qui peuvent agir comme un révélateur d'éléments dissimulés dans la vie quotidienne des organisations, dû à son pouvoir de transformation ${ }^{2}$.

Ces dernières décennies, les études concernant les crises ont pris des chemins différents selon leurs définitions ${ }^{3}$, ce qui a aussi influencé la façon de les analyser. Par leur caractère multiforme, les crises

\footnotetext{
${ }^{1}$ MORIN, E. (1976). Pour une crisologie, Communications. n 25, p. $149-163$.

${ }^{2}$ MORIN, E. (1994). Sociologie. Paris, Seuil.

${ }^{3}$ HERMANN, C. F. (1963). Some consequences of crisis which limit the viability of organizations. Administrative Science Quarterly, $\mathrm{n}^{\circ} 8$, p. $61-68$.
} 
concernent les domaines technologiques, sociaux, politiques, réglementaires, financiers, commerciaux, corporatifs ou écologiques.

Le concept de crise est une notion fortement liée à la perception des acteurs concernés, ce qui fait qu'une même situation peut être perçue (et définie) de différentes manières. En raison de leur caractère protéiforme, les cas de crise sont toujours uniques dans leurs caractéristiques et ils exigent des actions spécifiques.

\section{CONCEPT DE STRATEGIE ET DE MANAGEMENT}

\section{Stratégie}

Le concept de stratégie tel que nous l'entendons ici, est celui de M. Porter.

- pour M. Porter $(1980,1985)$ qui considère la stratégie comme une «ligne d'ensemble indiquant comment une entreprise va entrer en lice contre ses concurrents »;

- du point de vue systémique IVINZA cite Jacques Mélèse ${ }^{4}$ (1979), qui lui s'emploie plutôt à montrer que la «notion de stratégie provient des concepts systémiques et qu'une réelle analyse stratégique est en fait une analyse de système. ${ }^{5}$ Il définit la stratégie comme une « logique qui s'exprime par un ensemble de règles fixant un cadre et des contraintes pour les actions stratégiques présentes et futures, de manière à prendre en compte les interactions entre elles et avec les divers environnements ainsi que les effets dynamiques à délais variables. $»^{6}$.

Une stratégie est typiquement constituée des éléments suivants :

- mission et vision définissant les buts de l'entreprise ;

- orientations stratégiques, selon lesquelles l'entreprise se développe et souhaite investir ses ressources dans un horizon temporel défini ;

- formulation du business model, comment l'entreprise se positionne sur le marché et crée de la valeur ;

- buts mesurables, selon lesquels la mise en œuvre peut être vérifiée (souvent de nature financière, mais ne doivent pas y être restreints).

La plupart des organisations évoluent au sein d'un environnement complexe, marqué par une forte incertitude : les changements y sont discontinus ${ }^{7}$, la qualité de l'information imprécise et les interdépendances entre les acteurs instables. Dans de telles conditions, le stratège peut éprouver certaines difficultés à anticiper les évolutions qui pourront affecter l'organisation et à agir afin d'en absorber les effets. Figure centrale du management stratégique, le stratège évoque une catégorie d'acteurs qui contribue à la définition de la stratégie dans l'organisation.

\section{Management}

Même si le terme management nous vient de la langue anglaise, l'Académie Française l'a déjà inclut dans notre langue. Cependant la définition du terme est relativement vaste et englobe l'ensemble des techniques d'organisation de ressources (financières, humaines, matérielles...) mises en œuvre pour la gestion et l'administration d'une organisation (entreprise, association, administration...). ${ }^{8}$

\footnotetext{
${ }^{4}$ IVINZA LEPAPA, A. (2017). Informatique Stratégique : Cadre théorique et Applications dans les entreprises congolaises. Ed. Bookelis, Paris, page 45.

${ }^{5}$ MELESE, J. (1979). Approche systémique des organisations, Hommes et Techniques. Suresnes, page 58.

${ }^{6}$ MELESE, J. (1979). op. cit., page 60.

${ }^{7}$ EISENHARDT, K. M. (1989). Building theory from case study research. Academy of Management Review, vol. 14, no. 4, $532-550$.

${ }^{8}$ https://www.petite-entreprise.net/P-1660-81-G1-le-management-qu-est-ce-que-c-est.html
} 
Cela englobe l'art de diriger des individus afin d'obtenir une performance satisfaisante en termes de rendement, de productivité et de synergie des moyens matériels et des ressources humaines.

Dans un souci d'optimisation, il tend à respecter les intérêts et représentations des parties prenantes de l'entreprise. Afin de prendre en compte le temps, le risque et l'information sur les prises de décision de gestion, il est d'usage de distinguer ${ }^{9}$ :

- le management stratégique qui concerne la gestion du marché par la stratégie (c'est aussi une vision externe de la gestion);

- le management opérationnel qui concerne la gestion des processus propres à l'entreprise (c'est une vision plus interne centrée sur l'organisation);

- le contrôle de gestion tend à faire le lien entre ces deux types de management du fait de son positionnement au sein de l'entité.

Les enjeux du management sont de :

- diriger efficacement les ressources de l'organisation ;

- cela nécessite l'utilisation d'outils qui se révèlent faire appel particulièrement à l'économie, mais aussi à la sociologie et la psychologie. Ainsi le management doit réussir à prendre en compte les représentations et intérêts des différentes parties prenantes de l'organisation tout en restant dans une idée d'optimisation organisationnelle.

\section{MODÈLE DE FORMULATION DU MANAGEMENT STRATÉGIQUE}

On formule une stratégie en intégrant les opportunités et les contraintes de l'environnement externe avec les capacités et les ressources de l'organisation (environnement interne). La stratégie peut se résumer à trois questions de la figure 1.

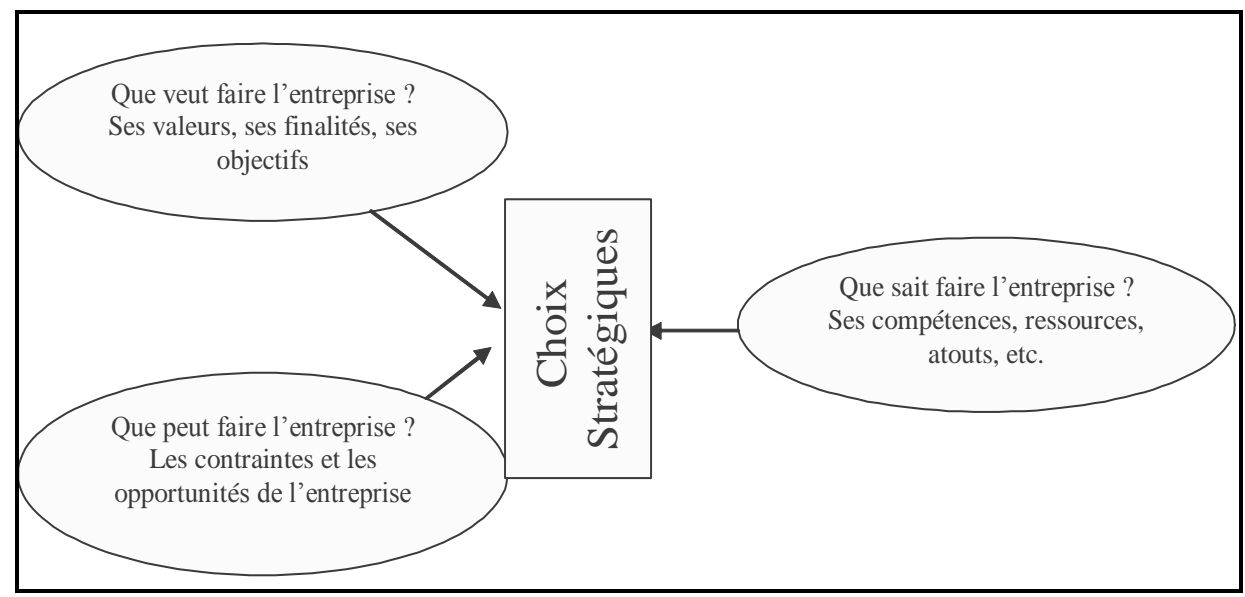

Figure 1. Mécanisme de base de la stratégie. Le schéma de la formulation Stratégique de Igor Ansoff

La stratégie d'une entreprise défini le positionnement commercial de l'entreprise par rapport à ses concurrents, ce qui sera mis en œuvre par la structure de l'entreprise. Le premier modèle général de la formulation de la stratégie a été mis au point par quatre professeurs de Harwad Business School : Learned, Christensen, Andrews et Guth en 1965 dans leur livre «Business Policy - Texts and Cases ».

\footnotetext{
${ }^{9}$ DAIGNE, J. F. (1991). Management en période de crise. Aspects stratégiques, financiers et sociaux. Paris, Les Éditions d'organisation.
} 
Ce modèle appelé LCAG du nom de ses auteurs est devenu une norme de facto pour plusieurs entreprises. Ce modèle est aussi appelé en anglais SWOT (Strengths, Weaknesses, Opportunities and Trheats) en français MOFF (Menaces, Opportunités, Forces et Faiblesses). ${ }^{10}$

Le management stratégique de l'information désigne l'utilisation de l'information à des fins stratégiques dans le but d'en tirer un avantage pour le fonctionnement et la prospérité de l'entreprise. ${ }^{11}$

La fonction de management stratégique concerne un "management d'anticipation" qui considère l'information comme une ressource vitale de l'entreprise "au même titre que l'argent, les matières premières ou le travail." 12

L'Information vitale dont il est question se partage en deux catégories :

- celle qui informe l'entreprise sur elle-même et sa propre évolution ;

- celle qui informe l'entreprise sur son environnement et sur son évolution. ${ }^{13}$.

\section{Les technologies de l'information et de communication dans la gestion des entreprises}

$\mathrm{Au}$ début des années 60, l'ordinateur fit son entrée dans les organisations pour soulager les gestionnaires dans le traitement de l'information et autres processus de gestion, etc...

"Dès le début des années 70, la complexité grandissante des technologies de l'information, alliée à la dépendance accrue des entreprises envers celles-ci, oblige ces entreprises à rechercher un plus haut niveau d'intégration entre le développement des systèmes d'information et leur planification stratégique ;.... Ces deux constats amènent chez le gestionnaire un changement de vision au niveau de la conception et au niveau de l'utilisation des systèmes d'information pour la gestion des entreprises." "14

Pour IVINZA LEPAPA, les technologies de l'information dans la gestion des entreprises ont été influencées par deux courants de pensée contemporains en management et en économie. Les deux courants proviennent de Harvard Business School. ${ }^{15}$

Le premier courant de pensée est d'origine managériale. Il est basé sur ce qu'on appelle aujourd'hui le paradigme d'Anthony. Ce courant de pensée a engendré, à partir de 1971, ce qu'on appelle l'approche classique et traditionnelle de l'informatique, qui est caractérisée par une vision interne de l'entreprise. ${ }^{16}$

Le second courant est basé sur les travaux de Michael PORTER. Ce courant de pensée a engendré à partir de 1985 avec Wiseman, ce qu'on appelle l'informatique stratégique. Cette informatique est caractérisée par une vision qui inclut les aspects internes et externes de l'entreprise.

Les Technologies de l'Information et de la Communication (TIC) peuvent être perçues comme des outils d'aide à la décision et à l'action. Leurs potentialités informationnelles et communicationnelles facilitent l'accès à un volume important d'information et sont censées influencer les capacités d'adaptation et de réactivité des stratèges.

Un nombre croissant d'organisations évolue au sein d'environnements complexes, marqués par une forte incertitude. Dans de telles conditions, le stratège peut éprouver certaines difficultés à anticiper les

10 MAYRHOFER, U. (2007). Management stratégique. Lexifac Gestion, Bréal, pages 38 - 39.

${ }^{11}$ PATEYRON, E. (1998). La Veille Stratégique. Economica, Paris, page 15.

12 LESCA, H. (1986). Système d'information pour le management stratégique de l'entreprise, L'entreprise intelligente. McGRAW-HILL, page 137.

13 TREMBLAY, C. (1992). L'informatique du Décideur, Pour intégrer l'informatique à la stratégie de l'entreprise. Marabout, Alleur, Belgique, page 74.

${ }^{14}$ LEVESQUE, G. \& SHEEHY, G. (1991). Les technologies de l'information pour la gestion des entreprises : un changement de vision. TIS, vol. $3 \mathrm{n}^{\circ} 2-3$, page 88.

15 IVINZA LEPAPA, A. (2007). Analyse de l'introduction de l'EDI dans les entreprises congolaises une contribution à l'impact organisationnel des TI tome 1: Concepts de base et cadre d'analyse théorique, Editions universitaires européennes, Allemagne.

${ }^{16}$ WISEMAN, C. (1987). L'informatique stratégique : nouvel atout de la compétitivité. Ed. d'organisation. Paris, pages $187-$ 207. 
évolutions qui pourront affecter l'organisation et à agir afin d'en absorber les effets. Le stratège évoque une catégorie d'acteurs qui contribue à la définition de la stratégie en influençant la construction des pratiques stratégiques. Face à la complexité de leur contexte d'action, les stratèges peuvent percevoir les Technologies de l'Information et de la Communication (TIC) comme des outils d'aide à l'anticipation.

\section{Adoption des technologies de l'information et de communication dans une entreprise}

Pour IVINZA LEPAPA, le modèle le plus utilisé aujourd'hui pour évaluer l'évolution de l'adoption des TI est SAM (strategic alignement model) modèle d'alignement stratégique de Venkatraman ${ }^{17}$. Selon lui l'approche économique des applications informatiques, télématiques, a conduit à travers la théorie des coups stratégiques à comprendre les applications et les services télématiques concurrentiels en gestion.

Depuis bientôt trois décennies les entreprises dites intelligentes organisent une fonction de management stratégique qui consiste à «Une démarche au travers de la quelle une entreprise, une organisation ou un système d'action collectif tente de trouver un équilibre satisfaisant entre des exigences (de compétitivité, de sécurité et de légitimité) partiellement contradictoire ${ }^{18}$

Il va sans dire que le système d'information pour le management stratégique devra recouvrir l'information vitale dont l'entreprise aura besoin pour diminuer son incertitude et dès lors garder son avance technologique.

Cette information, peut être interne et externe. Elle est Interne, parce qu'elle informe l'entreprise sur elle-même et sur sa propre évolution. Elle est Externe, parce qu'elle informe l'entreprise sur son environnement.

\section{Utilisation des TIC dans les entreprises Congolaises}

L'intégration des Technologies de l'Information et de Communication dans les entreprises, s'apprécie à travers leur système d'information et l'utilisation des outils technologiques. Armand (1992) définit le système d'information comme est un processus qui consiste à la création, à la collecte, traitement, stockage et à la communication de l'information.

Nous entendons par outil informatique, non seulement les ordinateurs qui servent à traiter les informations, mais aussi les réseaux qui les relient et permettent aux différents acteurs de coordonner leurs activités au sein de l'entreprise. Dans ce cas, nous sommes en présence d'une interconnexion de machines. Everaere (1997) stipule qu'ici, l'idée de relier est essentiellement pour la flexibilité ; car la capacité d'adaptation à des aléas imprévisibles dans un contexte d'urgence est basée sur un échange rapide d'informations.

Les entreprises congolaises ne dépassent pas l'étape de la formulation de stratégie.

Le problème de ces entreprises c'est la mise en œuvre des systèmes d'information stratégique (SIS).

D'abord, le management doit être conscient que la mise en œuvre d'une stratégie implique de profonds bouleversements. Les employés doivent porter le processus de changement. C'est pourquoi une attention particulière doit être apportée à la conduite du changement, qui est souvent oubliée dans le cadre de projets dans le domaine des TIC.

L'instrument le plus important pour une mise en œuvre réussie d'une stratégie est le portefeuille stratégique que les entreprises congolaises ignorent. Dans ce dernier, des projets concrets sont dérivés de la stratégie en tant qu'initiatives stratégiques. Les TIC font aujourd'hui partie de tous les grands projets et représentent une partie conséquente des coûts, de la complexité et des risques de ces initiatives stratégiques. Ainsi, une compétence correspondante pour la formulation des mandats de projets est une condition obligatoire.

\footnotetext{
17 IVINZA LEPAPA, A. (2017). Informatique Stratégique: Cadre théorique et Applications dans les entreprises congolaises. Ed. Bookelis, Paris, page 35.

${ }^{18}$ ANTHONY R. N., op.cit. page 15.
} 


\section{Identification des TIC comme facteurs de certaines crises managériales}

L'identification et la classification des crises visent ainsi à offrir aux gestionnaires un cadre de référence pour leur jugement ${ }^{19}$ et pour les aider à repérer de telles situations, même s'il s'agit d'un ${ }^{20}$ phénomène incertain et évolutif.

Pour Reix (2000), les TIC se caractérisent par des capacités de stockage, de traitement et de communication sans commune mesure avec les anciennes technologies ${ }^{21}$. Ainsi, il existerait une sorte de "saut technologique ou leapfrog technologique", impliquant des gains de productivité et une plus grande efficacité des systèmes de gestion. Un tel progrès est difficilement mesurable, notamment pour des technologies associées aux fonctions de support, telles le contrôle de gestion. Cependant, il semble effectivement que les TIC, en se perfectionnant du point de vue technique, ouvrent des opportunités en matière de contrôle.

Parmi ces technologies, développées durant les années 80-90, les systèmes client-serveurs constituent un progrès notable. Ils permettent d'avoir à la fois un système centralisé (assurant une certaine sécurité des données) et réparti selon les besoins des utilisateurs. En termes d'applications, pour le contrôle de gestion, les systèmes de gestion de base de données (SGBD) multidimensionnels marquent un saut technologique semblable. Ils permettent l'exploitation de bases de données partagées, avec un langage unifié et offrent des possibilités d'extraction de données riches. Les différents SGBD de l'entreprise peuvent disposer d'interfaces entre eux, mais l'intégration n'est pas forcément automatique.

En définitive, il faut donc comprendre les TIC, en insistant sur l'amélioration du partage de l'information dans l'organisation, relativement aux systèmes décentralisés des années 70, caractérisés par une certaine anarchie avec des applications locales, et des données non homogènes difficilement partageables. Dans ce sens, le développement de systèmes d'information intégrés, ouvre de nouvelles opportunités en matière d'organisation générale et de coordination par la fonction contrôle de gestion.

\section{L'APPROCHE STRATÉGIQUE DES TIC DANS LES ENTREPRISES EN AFRIQUE ET EN RD CONGO}

L'Afrique vit globalement une pleine croissance qui semble inarrêtable. Malgré les incidents de parcours (prix des matières premières...) et certaines tensions politiques, tous les facteurs sont en place.

L'urbanisation en Afrique, ce sont 20 à 25 millions de nouveaux urbains chaque année, ce qui permet l'émergence d'une classe moyenne. La multiplication des entreprises conduit à une croissance exponentielle des services : conseil, financement, recrutement, formation, gardiennage.

L'appropriation et le développement local de techniques adaptées et des technologies les plus modernes, permettent à l'Afrique de sauter des étapes (le fameux « leapfrog », littéralement « saut de grenouille »), en faisant l'économie de coûteux réseaux fixes de téléphone ou de distribution d'électricité. ${ }^{22}$

Plus récemment, le concept de saut est utilisé dans le contexte du développement durable pour les pays en développement en tant que théorie du développement qui peut accélérer le développement en ignorant des technologies et des industries inférieures, moins efficaces, plus coûteuses ou plus polluantes et se dirigeant directement vers des technologies plus avancées. ${ }^{23}$

Le concept de saut de grenouille a été utilisé à l'origine dans le contexte des théories de la croissance économique ${ }^{24}$ et des études d'innovation dans l'organisation industrielle avec un accent particulier sur

\footnotetext{
${ }^{19}$ LAGADEC, P. (1995). Cellules de Crise : Les conditions d'une conduite efficace. Paris, Editions d'organisation.

${ }^{20}$ THIÉTART, R. A. \& FORGUES, B. (1997). Action, structure and chaos. Organization Studies, vol. 18, n 1, p. 119 - 143.

${ }^{21}$ REIX, R. (2000). Systèmes d'information et management des organisations. 3e ed., Paris, Vuibert

${ }^{22}$ MAKTHAR DIOP : http://www.banquemondiale.org/fr/news/opinion/2017/10/11/africa-can-enjoy-leapfrog-development

${ }^{23} \mathrm{https}: / /$ ducalingo.com/fr/dic-en/leapfrogging

${ }^{24}$ KOLTER, P. \& DUBOIS, B. (1997). Marketing management. 9e édit. Publi-Union.
} 
la concurrence entre les entreprises. Il est basé sur la notion de «gales de destruction créative» de Joseph Schumpeter. L'hypothèse propose que les entreprises détenant des monopoles basées sur les technologies historiques soient moins incitées à innover que les concurrents potentiels et, par conséquent, elles finissent par perdre leur rôle de leadership technologique lorsque de nouvelles innovations technologiques radicales sont adoptées par de nouvelles entreprises qui sont prêtes à prendre les risques. Lorsque les innovations radicales deviennent éventuellement le nouveau paradigme technologique, les entreprises nouvelles entrent en colère devant les premières entreprises de premier plan.

Le mobile banking ${ }^{25}$ est peut-être l'un des cas les plus notables du saut technologique africain, surnommé «leapfrog ». Les transferts de compétences ne sont en effet plus linéaires, du Nord vers le Sud. Aujourd'hui, $70 \%$ des Congolais utilisent leur smartphone pour payer leurs factures, envoyer de l'argent à leurs proches, soit $60 \%$ du volume global des transactions effectuées dans le pays, ce qui en fait un élément structurant, d'après la Banque mondiale, de croissance inclusive. Depuis lors, des services mobiles ou numériques imaginés en Afrique et pour l'Afrique se sont multipliés, de Nairobi à Dakar en passant par Le Cap, Accra ou Kinshasa, révolutionnant le rapport aux infrastructures. L'écosystème numérique africain est aussi le reflet de l'histoire certes courte mais bien globale d'internet et des technologies numériques.

\section{CONCLUSION}

Dans cet article, nous avons essayé d'aborder certains facteurs de crise qui perturbent l'introduction des TIC dans les organisations. Pour nous congolais, il nous faut un apprentissage organisationnel circonscrit à des actions délibérées telles que la formation, la veille stratégique ou même le e-learning mais s'envisage davantage dans une approche systémique de l'organisation où les savoirs collectifs émergent à travers les pratiques et les expériences accumulées.

Les organisations suivant le paradigme fonctionnaliste des écoles systémiques et de contingence structurelle doivent continuer à survivre devant la turbulence de l'environnement et de l'évolution rapide des $\mathrm{TI}^{26}$. Ce qui implique, bien sûr, la maitrise des changements qui se répercuteront sur les organisations ; afin de bénéficier d'énormes occasions qu'offrent les Technologies de l'Information et de la Communication, les dirigeants soucieux du devenir de leurs affaires, doivent de plus en plus renforcer leurs actions dans tous les domaines de gestion de leur entreprise. Cette action doit être à la portée des cadres, des responsables des décisions tactiques et opérationnelles dans l'entreprise, afin de donner non seulement une grande quantité d'informations riches en qualité, pertinentes et fiables qui circulent aussi bien en amont qu'en aval au sein de l'entreprise comme dans son environnement extérieur. Une telle attitude vis à vis des TIC peut conférer aux entités économiques des pays en voies de développement, une performance globale (économique, financière, technique, commerciale et en ressources humaines) gage d'une compétitivité durable dans un environnement turbulent.

Les TIC élargissent les possibilités des pays en développement de participer aux marchés internationaux. Elles transforment radicalement les modalités de production, de livraison, de vente et d'achat de biens et services. Elles mettent en relation par la connectivité numérique, un nombre impressionnant de personnes et d'entreprises, prêtes à participer à l'économie du savoir et à y contribuer. Grâce à une accélération et un contrôle croissant des échanges, ces instruments permettent aux acteurs relativement défavorisés (les propriétaires des petites entreprises des pays en développement), d'avoir accès à l'information, à la communication et à un savoir qui étaient jadis hors de leur portée.

\footnotetext{
${ }^{25}$ IVINZA LEPAPA, A. (2017). Monétique et Transactions électroniques : concepts et principes de base. Ed. Bookelis, Paris, page 24

${ }^{26}$ IVINZA LEPAPA, A. op.cit., page 58.
} 


\section{RÉFÉRENCES}

DAIGNE, J. F. (1991). Management en période de crise. Aspects stratégiques, financiers et sociaux. Paris, Les Éditions d'organisation.

EISENHARDT, K. M. (1989). Building theory from case study research. Academy of Management Review, vol. 14, no. 4, $532-550$.

HERMANN, C. F. (1963). Some consequences of crisis which limit the viability of organizations. Administrative Science Quarterly, n 8, p. $61-68$.

IVINZA LEPAPA, A. (2017). Informatique Stratégique : Cadre théorique et Applications dans les entreprises congolaises. Ed. Bookelis, Paris, page 35.

IVINZA LEPAPA, A. (2017). Monétique et Transactions électroniques : concepts et principes de base. Ed. Bookelis, Paris, page 24.

IVINZA LEPAPA, A. (2007). Analyse de l'introduction de l'EDI dans les entreprises congolaises une contribution à l"impact organisationnel des TI tome 1: Concepts de base et cadre d'analyse théorique, Editions universitaires européennes, Allemagne.

KOLTER, P. \& DUBOIS, B. (1997). Marketing management. 9e édit. Publi-Union.

LAGADEC, P. (1995). Cellules de Crise : Les conditions d'une conduite efficace. Paris, Editions d'organisation.

LESCA, H. (1986). Système d'information pour le management stratégique de l'entreprise, L'entreprise intelligente. McGRAW-HILL, page 137.

LEVESQUE, G. \& SHEEHY, G. (1991). Les technologies de l'information pour la gestion des entreprises : un changement de vision. TIS, vol. $3 \mathrm{n}^{\circ} 2-3$, page 88.

MAYRHOFER, U. (2007). Management stratégique. Lexifac Gestion, Bréal, pages 38 - 39.

MELESE, J. (1979). Approche systémique des organisations, Hommes et Techniques. Suresnes, page 58 .

MORIN, E. (1976). Pour une crisologie, Communications. n 25, p. $149-163$.

MORIN, E. (1994). Sociologie. Paris, Seuil.

PATEYRON, E. (1998). La Veille Stratégique. Economica, Paris, page 15.

REIX, R. (2000). Systèmes d'information et management des organisations. 3e ed., Paris, Vuibert

THIÉTART, R. A. \& FORGUES, B. (1997). Action, structure and chaos. Organization Studies, vol. $18, \mathrm{n}^{\circ} 1$, p. $119-143$.

TREMBLAY, C. (1992). L'informatique du Décideur, Pour intégrer l'informatique à la stratégie de l'entreprise. Marabout, Alleur, Belgique, page 74.

WISEMAN, C. (1987). L'informatique stratégique: nouvel atout de la compétitivité. Ed. d'organisation. Paris, pages 187-207.

MAKTHAR DIOP : http://www.banquemondiale.org/fr/news/opinion/2017/10/11/africa-can-enjoyleapfrog-development

https://www.petite-entreprise.net/P-1660-81-G1-le-management-qu-est-ce-que-c-est.html

https://educalingo.com/fr/dic-en/leapfrogging 